\title{
Lumbosacral ependymomas: a review of the management of intradural and extradural tumors
}

\author{
Daniel R. Fassett, M.D., AND Meic H. Schmidt, M.D. \\ Department of Neurosurgery, University of Utah School of Medicine, Salt Lake City, Utah
}

\begin{abstract}
Object. The goal of this study was to review the management of intra- and extradural ependymomas. Spinal ependymomas most commonly occur as intramedullary tumors throughout the spinal axis. In the lumbosacral region, ependymomas are most commonly associated with the conus medullaris and cauda equina, but can also occur extradurally in the sacrum, presacral tissues, or subcutaneous tissues over the sacrum. These two tumor locations produce different management concerns. Intradural ependymomas, especially those in the lumbosacral region, are now recognized for their potential to spread throughout the central nervous system (CNS), whereas extradural tumors elicit more concern for their association with extraneural metastases.

Methods. The authors have reviewed the literature regarding both of these distinct tumors and have summarized recommendations for the management of intra- and extradural lumbosacral ependymomas. For both tumors, it appears that gross-total resection is the treatment of choice when feasible. The role of radiation therapy has not been adequately studied for either tumor location, but most clinicians use this modality in patients with subtotal resection of intradural ependymomas, local recurrence, or CNS dissemination. Data supporting the use of radiation therapy for extradural ependymomas are lacking. There does not appear to be a significant role for chemotherapy in either tumor location.

Conclusions. Despite the risk for local recurrence and CNS dissemination, the prognosis for intradural lumbosacral ependymomas is good, with a greater than $90 \%$ 10-year patient survival in most series. The prognosis for extradural ependymomas does not appear to be as good. Much depends on extradural tumor location, however; the outlook is better for dorsal sacral tumors than presacral tumors.
\end{abstract}

KEY WORDS • intradural ependymoma - extradural ependymoma • lumbosacral lesion

Spinal ependymomas are very rare tumors, with approximately 227 intradural spinal ependymomas diagnosed each year in the US. Of the intradural ependymomas, approximately $50 \%$ are intramedullary, above the conus, and the remaining 50\% occur in association with the terminal filum, cauda equina, and conus medullaris. ${ }^{3,6,22}$ In the lumbosacral region, ependymomas account for $90 \%$ of intradural tumors. ${ }^{10}$ In addition to occurring intradurally, ependymomas can also sometimes occur extradurally around or in the sacrum.

Spinal ependymomas are most common in the fourth decade of life and are more common in male patients. ${ }^{5,33,37}$ Sonneland, et al. ${ }^{32}$ reported a 1.7:1.0 male/female ratio and a mean age at onset of 36.4 years (range 6-82 years) in the largest series (77 patients) of myxopapillary ependymomas reported in the literature. All of the tumors were intradural in this series, with the majority occurring in the lumbosacral region.

Bimodal peaks have been suggested for extraspinal ependymomas in the sacral region, with one peak at less than 8 years of age and one in the fourth decade of life. ${ }^{20,21}$ Others have suggested that extraspinal ependymomas oc-

Abbreviations used in this paper: $\mathrm{CNS}=$ central nervous system; $\mathrm{CSF}=$ cerebrospinal fluid; $\mathrm{MR}=$ magnetic resonance. cur in younger patients compared with intraspinal ependymomas. ${ }^{1,15,17,21,25}$ There appears to be no significant sex predominance for extradural ependymomas. ${ }^{15,21,25}$

\section{OVERVIEW}

Tumor Locations and Features

Spinal ependymomas can originate from either the ependymal lining of the central canal of the spinal cord, ependymal cell clusters in the terminal filum, or from ependymal rests left during embryonic development. Intramedullary tumors above the lumbosacral region almost uniformly arise from the ependymal lining of the spinal cord. These intradural, intramedullary ependymomas are predominantly the cellular ependymoma histiotype. .,22 $^{6}$

In the lumbosacral region, the majority of ependymomas arise from the intradural terminal filum. These are characteristically well-encapsulated, sausage-shaped tumors with the nerve roots of the cauda equina draped over the surface of the lesion or enveloped by it (Fig. 1). Myxopapillary ependymomas comprise the majority of ependymomas that arise in this area, but papillary and cellular histiotypes can also occur here. ${ }^{3-5,9,10,13,22}$

Extradural ependymomas are very rare and are known to occur in four perisacral locations, as follows: 1) the 


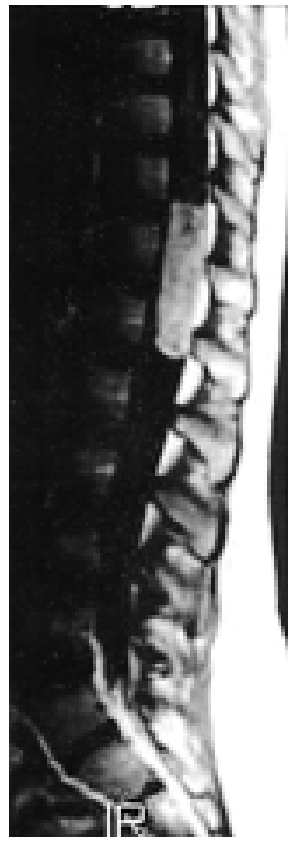

Fig. 1. Sagital MR image with Gd contrast demonstrating an intradural ependymoma at the level of the cauda equina.

extradural spinal canal in association with the dural part of the terminal filum; 2) the bone substance of the sacrum; 3 ) the pelvic cavity anterior to the sacrum; and 4) the subcutaneous tissues dorsal to the sacrum. ${ }^{10,14,17,20,21,30}$ Because of the erosive nature of these tumors, it may be impossible to differentiate between one that starts within the bone of the sacrum and one that starts in the extradural spinal canal and erodes into the sacrum. Based on the number of case reports in the literature, the posterior subcutaneous location is the most common one for extradural ependymomas, followed by the presacral region. . $^{111,25}$

Intraspinal extradural sacral ependymomas arise from ependymal cell remnants in the extradural dural part of the terminal filum. The other extradural ependymomas likely arise from ependymal rests that are present at the time of birth. Bale ${ }^{2}$ reported finding ependymal rests in the dorsal sacral subcutaneous tissues in necropsy samples obtained in 10 of 15 infants. Others have reported finding ependymal rests in tissue obtained during pilonidal cyst resection. ${ }^{25}$ One theory is that the ependymal cell rests arise from the coccygeal medullary vestige, a remnant of the dural part of the terminal filum that involutes during embryonic development. Others hypothesize that these ependymal rests are heterotopias that occur as a result of incomplete closure of the neural arch. ${ }^{12,16,17,20}$ Presacral ependymomas are thought to arise from ependymal cell rests or in association with cauda equina nerve roots that have exited the sacral neural foramen. Histologically, all reported cases of extradural ependymomas have been myxopapillary types. ${ }^{8,10,16,17,25,34}$

\section{Tumor Symptoms}

Back pain and occasionally radicular pain are the most common presenting symptoms for intradural ependymomas arising from the terminal filum in the lumbosacral re- gion. Motor or sensory abnormalities and bladder dysfunction may also develop in association with these lesions involving the cauda equina. ${ }^{5,24}$

Extraspinal ependymomas can present with a variety of symptoms caused by local mass effect. Intrasacral and intraspinal extradural tumors present most commonly with local pain caused by erosion into the sacrum, but can also present with radicular or neurological symptoms caused by neural element compression. ${ }^{2,24}$ Dorsal subcutaneous tumors typically present as a growing, asymptomatic mass in the intergluteal fold or buttocks. Occasionally there can be pain or tenderness caused by local mass effect, and very rarely neurological symptoms are reported. ${ }^{1,9,17,21,25}$ Presacral pelvic masses can grow to be very large, because of significant tolerance for masses in this area. With sufficient size, presacral masses eventually cause bowel or bladder dysfunction from local mass effect or neural involvement (Fig. 2). 2,10,21 Some patients may have a palpable mass on rectal examination. Rarely, presacral tumors cause pain in a sciatic nerve distribution or local pain from erosion into the sacrum. ${ }^{2,20,21,34}$

\section{Preoperative Evaluation}

All patients with these tumors require a complete neurological examination and MR imaging to delineate local disease. Intradural ependymomas can disseminate through CSF pathways throughout the CNS, but are not likely to metastasize outside it, ${ }^{21}$ although rare cases of this phenomenon have been reported. ${ }^{27,35}$ Therefore, we recommend preoperative screening MR imaging of the entire CNS to rule out tumor dissemination. Screening of other organ systems for metastases is not needed for intradural tumors. Preoperative plain anteroposterior and lateral $\mathrm{x}$-ray films of the lumbosacral spine may also be valuable for comparison if instability or deformity develop after laminectomy surgery. ${ }^{23}$

Extradural tumors, in contrast, are not likely to disseminate within the CNS, but are at significant risk of metastasizing to other organ systems, such as lymph, bone, lung, and liver. Use of MR or computerized tomography studies is sufficient to evaluate local disease and we see no need to screen the entire CNS. In addition, plain x-ray films or computerized tomography scans may be warranted in extradural ependymomas to provide further detail
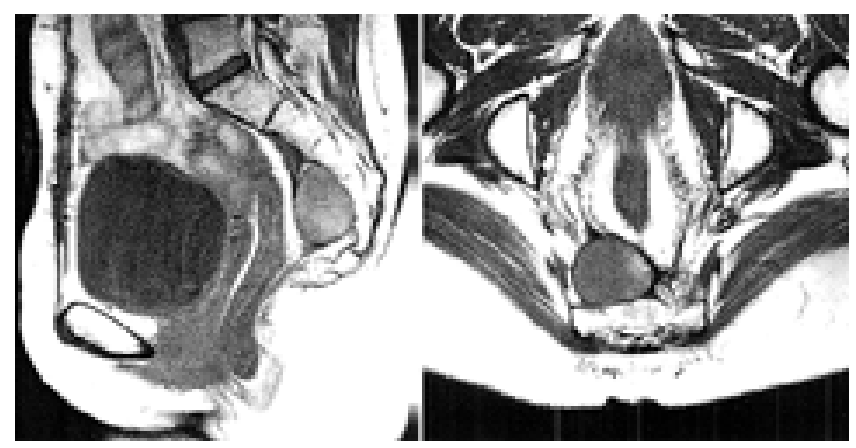

Fig. 2. Left: Sagittal noncontrast MR image demonstrating a presacral ependymoma and an enlarged bladder due to urinary retention. Right: Axial noncontrast MR image demonstrating a presacral ependymoma. 
about bone involvement. We recommend paying particular attention to general examination for lymphadenopathy, especially in the inguinal lymph nodes,$^{15}$ and would also obtain chest x-ray films, liver function tests, and alkaline phosphatase levels preoperatively. Presacral tumors warrant a special workup that likely requires an abdominal/ pelvic surgical specialist. Other studies, such as barium enema, intravenous pyelogram, cystoscopy, and pelvic examination may need to be performed for presacral tumors. ${ }^{21}$

\section{Treatment Options}

There are no prospective, randomized studies in which the management options of surgery alone, surgery followed by radiation, radiation alone, or chemotherapy are examined.

Surgery Alone. The surgical goal with all ependymomas, whether they are intra- or extradural, is gross-total resection when feasible. ${ }^{16}$ Gross-total resection provides a possibility for cure without the definite need for adjuvant therapy. For intradural ependymomas in this location, gross-total resection is the most frequently cited factor influencing prognosis in terms of local recurrence and patient survival. ${ }^{5,28,33}$ Some authors report higher recurrence rates for tumors removed in a piecemeal fashion, even though gross-total resection was achieved..$^{33,35}$ Therefore, when possible, en bloc resection is preferred over piecemeal removal. Other factors, such as duration of clinical symptoms and tumor involvement with conus medullaris or cauda equina, have also been cited (Fig. 3). ${ }^{5}$

Obviously, when the conus medullaris and cauda equina are involved with the tumor, achieving gross-total resection becomes more difficult. In fact, Celli, et al., ${ }^{5}$ found that gross-total resection could be obtained in only $43 \%$ of cases when the conus medullaris or cauda were involved with the tumor. They also noted that, despite gross-total resection at the time of surgery, tumors involving the conus medullaris or cauda equina were more likely to recur. Sonneland, et al., ${ }^{33}$ reported gross-total resection in $59 \%$ of a series of 77 patients with predominantly terminal filum/conus medullaris/cauda equina lesions.

For posterior extradural lesions without sacral involvement, wide local excision for gross-total resection is advo-

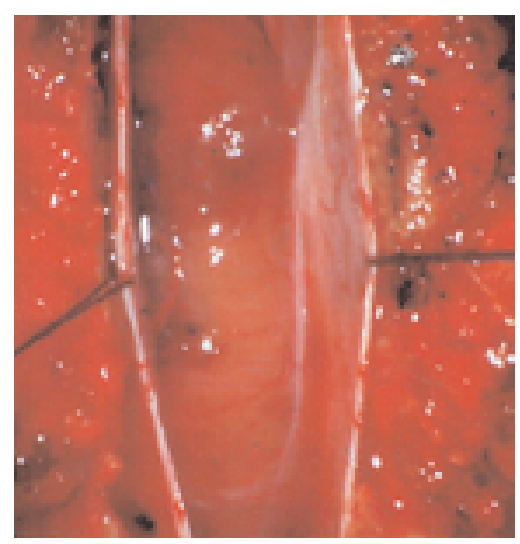

Fig. 3. Intraoperative photograph of an intradural ependymoma in the lumbar thecal sac. cated. ${ }^{10,20}$ Plastic surgery assistance may be needed for complex skin closure techniques required after resection of large masses. Some authors have recommended coccygectomy in addition to local excision as a means of reducing the risk of recurrence. ${ }^{15}$

The surgical approach for presacral lesions depends on the degree of sacral involvement. Isolated presacral tumors with minimal to no sacral involvement may be radically resected via just an anterior approach. When the sacrum is involved, combined anterior-posterior approaches, either in the same sitting or during different operations, may be needed. ${ }^{10,20}$

Resection Followed by Radiation. The role of radiation for lumbosacral ependymomas has not been adequately studied to warrant firm conclusions. There appears to be a consensus for radiation therapy in cases of subtotal resections of intradural tumors, although the role for radiation in cases of gross-total resection and extradural ependymomas is controversial. $3,5,7,10,11,16,28,29,31,33,35$

Sonneland, et al., ${ }^{33}$ based on their large series of patients, recommended local radiation therapy in cases of subtotal or piecemeal gross-total resection. Nevertheless, in reviewing their data, there appears to be no trend toward improved survival or recurrence rates with radiation therapy. Ross, et al. ${ }^{28}$ in a review of the 131 cases in the literature, concluded that although no differences in outcome after radiation have been shown for gross-total and subtotal resection, results in individual cases show that long-term tumor control can be achieved with this modality.

Waldron, et al., ${ }^{35}$ in a large series of spinal ependymomas, performed multivariate analysis in patients who received radiation after surgery. They evaluated the following variables: age, sex, preoperative symptom duration, preoperative functional status, tumor location, and histological grade. They found that only histological grade was statistically significant in regard to predicting recurrencefree survival and cause-specific survival. They reported a cause-specific actuarial survival at 5 and 10 years of 86 and $81 \%$, respectively. Nevertheless, their results may not correspond completely with lumbosacral ependymomas because in their group of 59 patients approximately half of the tumors were above the level of the conus medullaris. They note that in patients with well-differentiated tumors, like those typically found in the lumbosacral region, there was a 97\% 5-year, cause-specific survival. Although these survival rates with radiation appear to be excellent, when we compare these figures with overall survival rates for lumbosacral ependymomas there appears to be no significant difference. Waldron, et al. ${ }^{35}$ reported no cases of radiation myelopathy in the 59 spinal ependymomas treated, and others report very low complication rates with spinal irradiation in this area. ${ }^{31}$ Therefore, although they are not scientifically proven, the potential benefits of radiation therapy appear to outweigh the risks and can be justified in cases of subtotal tumor resection.

Radiation Therapy After Biopsy Sampling. Waldron, et al. ${ }^{35}$ report on five patients who received radiation therapy after biopsy sampling in which a diagnosis of ependymoma was made. This subgroup was included in a larger series of 59 patients who received radiation after surgery (biopsy or resection), and it is difficult to discern from the 
authors' data the tumor location, histological findings, and results in these five patients. Waldron, et al., report that three of these five patients were alive at the last followup review. Others have used radiation therapy alone after biopsy procedures for lumbosacral ependymomas, with apparently very good long-term results, although the number of cases in each series is small. ${ }^{32,36}$

Radiation Therapy for Recurrence or Metastases. For intradural ependymomas, radiation therapy is often used in cases of local recurrence and disseminated disease within the CNS. Intradural myxopapillary ependymomas have been shown to be radiosensitive, and long-term tumor control can be achieved with this adjunctive therapy in most cases of local recurrence or CNS metastases. ${ }^{7,28}$

Extradural ependymomas have not shown as promising results as intradural tumors. These extradural tumors are rare; therefore, sufficient data are lacking to draw conclusions on the effectiveness of radiotherapy. Most cases of local recurrence are treated with surgery and not radiation. With the limited data available, prolonged survival has not been observed in patients who receive radiotherapy for extradural ependymomas. Metastatic disease to other organ systems typically does not respond to radiation therapy or chemotherapy. ${ }^{15}$

Chemotherapy. This modality has been restricted primarily to patients with recurrent disease that has been refractory to resection and radiation. A number of chemotherapeutic agents have been tried in small series of recurrent spinal ependymomas, but no studies have shown compelling evidence to indicate that chemotherapy may be a primary treatment for these tumors. ${ }^{6,10,33,34}$ Individual case reports ${ }^{18}$ describe control of myxopapillary ependymoma with chemotherapy, but sufficient data are lacking to draw any significant conclusions regarding this regimen.

Prognosis for Ependymomas. Most ependymomas occurring in the lumbosacral region are myxopapillary on histological examination. Even though intraspinal myxopapillary ependymomas are considered benign, they are known to recur locally and can disseminate through CSF pathways. Even with gross-total resection, recurrence rates of 4 to $29 \%$ have been reported. ${ }^{3-5}$ Although described by Davis and Barnard ${ }^{9}$ in 1985, the potential for dissemination of these lesions throughout the CNS has only recently been truly appreciated. ${ }^{26,27,38}$ Disseminated disease can arise many years after initial tumor presentation. ${ }^{9,26}$

Despite the significant risk of local recurrence and CSF dissemination, the overall prognosis for lumbosacral intradural myxopapillary ependymomas appears to be very good. Sonneland, et al. ${ }^{33}$ reported long-term survival in approximately $95 \%$ of patients in their series of 77 patients. Mork and Loken, ${ }^{22}$ in another large series of spinal ependymomas, reported a 10 -year survival rate of 94\% for patients with lumbosacral intradural ependymomas and noted that the myxopapillary histiotype had a favorable prognosis. The few patients who die as a result of primary disease typically do so after a prolonged course with multiple recurrences.

Extradural ependymomas are a different entity compared with intradural tumors because of the risk of local recurrence and distal metastasis. For presacral tumors, lo- cal recurrence has been reported to be as high as $60 \%$, with mortality rates as high as $75 \%$ over a period of 4 years for cases of recurrence. ${ }^{20,34}$ Local recurrence for dorsal subcutaneous tumors is approximately $25 \%$ at 15 years. ${ }^{10}$ Overall, extradural lesions are much more likely to metastasize to other organ systems than intradural tumors, with the lymphatic system, lungs, bones, and liver being the most common sites. ${ }^{9}$ The tendency for extradural tumors to metastasize is likely because of the closer proximity of lymphatic and vascular channels in extradural locations. The risk of metastases also depends on the specific extradural location, with dorsal subcutaneous tumors at greater risk for metastasis than presacral tumors. Intrasacral lesions have also been reported to metastasize, but this appears to be very rare. ${ }^{19,27}$ Metastases have been reported in up to $20 \%$ of dorsal subcutaneous tumors in long-term follow up. ${ }^{14}$ Metastatic disease from extradural ependymomas appears to carry a grim prognosis due to lack of response to adjuvant therapies.

\section{CONCLUSIONS}

Lumbosacral ependymomas consist of two distinct entities that must be managed differently. The more common intradural ependymoma has a significant risk of both local recurrence and dissemination to other areas of the CNS. The rare extradural sacral region ependymoma has the potential for extraneural metastases in addition to local recurrence. The possibility of metastases for lesions in both of these locations should be taken into account in preoperative evaluation and in long-term follow up.

In both tumor locations, gross-total resection is the treatment of choice when feasible. Radiation therapy may be valuable for subtotal intradural resections or CNS metastases from intradural tumors. Radiotherapy for extradural lesions does not appear to be as effective and is more controversial.

\section{References}

1. Anderson MS: Myxopapillary ependymomas presenting in the soft tissue over the sacrococcygeal region. Cancer 19: 585-590, 1966

2. Bale PM: Ependymal rests and subcutaneous sacrococcygeal ependymoma. Pathology 12:237-243, 1980

3. Bavbek M, Altinors MN, Caner HH, et al: Lumbar myxopapillary ependymoma mimicking neurofibroma. Spinal Cord 39: 449-452, 2001

4. Burtscher J, Felber S, Twerdy K, et al: Endoscope-assisted interlaminar removal of an ependymoma of the cauda equina. Minim Invasive Neurosurg 45:41-44, 2002

5. Celli P, Cervoni L, Cantore G: Ependymoma of the filum terminale: treatment and prognostic factors in a series of 28 cases. Acta Neurochir 124:99-103, 1993

6. Chamberlain MC: Salvage chemotherapy for recurrent spinal cord ependymoma. Cancer 95:997-1002, 2002

7. Chinn DM, Donaldson SS, Dahl GV, et al: Management of children with metastatic spinal myxopapillary ependymoma using craniospinal irradiation. Med Pediatr Oncol 35:443-445, 2000

8. Chou S, Soucy P, Carpenter B: Extraspinal ependymoma. J Pediatr Surg 22:802-803, 1987

9. Davis C, Barnard RO: Malignant behavior of myxopapillary ependymoma. Report of three cases. J Neurosurg 62:925-929, 1985 


\section{Review of the management of lumbosacral ependymomas}

10. Fourney DR, Fuller GN, Gokaslan ZL: Intraspinal extradural myxopapillary ependymoma of the sacrum arising from the filum terminale externa. Case report. J Neurosurg (Spine 2) 93: 322-326, 2000

11. Gerston KF, Suprun H, Cohen H, et al: Presacral myxopapillary ependymoma presenting as an abdominal mass in a child. J Pediatr Surg 20:276-278, 1985

12. Gregorios JB, Green B, Page L, et al: Spinal cord tumors presenting with neural tube defects. Neurosurgery 19:962-966, 1986

13. Hallacq P, Labrousse F, Streichenberger N, et al: Bifocal myxopapillary ependymoma of the terminal filum: the end of a spectrum? J Neurosurg (Spine 3) 98:288-289, 2003

14. Helwig EB, Stern JB: Subcutaneous sacrococcygeal myxopapillary ependymoma. A clinicopathologic study of 32 cases. Am J Clin Pathol 81:156-161, 1984

15. Kramer GW, Rutten E, Sloof J: Subcutaneous sacrococcygeal ependymoma with inguinal lymph node metastasis. J Neurosurg 68:474-477, 1988

16. Lemberger A, Stein M, Doron J, et al: Sacrococcygeal extradural ependymoma. Cancer 64:1156-1159, 1989

17. Lynch J, Kelly N, Fitzpatrick B, et al: A sacrococcygeal extraspinal ependymoma in a 67-year-old man: a case report and review of the literature. Br J Plast Surg 55:80-82, 2002

18. Madden JR, Fenton LZ, Weil M, et al: Experience with tamoxifen/etoposide in the treatment of a child with myxopapillary ependymoma. Med Pediatr Oncol 37:67-69, 2001

19. Miralbell R, Louis DN, O'Keeffe D, et al: Metastatic ependymoma of the sacrum. Cancer 65:2353-2355, 1990

20. Morantz RA: Ectopic ependymoma of the sacrococcygeal region, in Doty JR, Rengachary SS (eds): Surgical Disorders of the Sacrum. New York: Theime Medical, 1992, pp 177-179

21. Morantz RA, Kepes JJ, Batnitzky S, et al: Extraspinal ependymomas. J Neurosurg 51:383-391, 1979

22. Mork SJ, Loken AC: Ependymoma: a follow-up study of 101 cases. Cancer 40:907-915, 1977

23. Papagelopoulos PJ, Peterson HA, Ebersold MJ, et al: Spinal column deformity and instability after lumbar or thoracolumbar laminectomy for intraspinal tumors in children and young adults. Spine 22:442-451, 1997

24. Post KD, McCormick PC: Intrasacral ependymoma, in Doty JR, Rengachary SS (eds): Surgical Disorders of the Sacrum. New York: Theime Medical, 1992, pp 181-183

25. Pulitzer DR, Martin PC, Collins PC, et al: Subcutaneous sacrococcygeal ("myxopappillary") ependymal rests. Am J Surg Pathol 12:672-677, 1988
26. Rezai AR, Woo HH, Lee M, et al: Disseminated ependymomas of the central nervous system. J Neurosurg 85:618-624, 1996

27. Rickert CH, Kedziora O, Gullotta F: Ependymoma of the cauda equina. Acta Neurochir 141:781-782, 1999

28. Ross DA, McKeever PE, Sandler HM, et al: Myxopapillary ependymoma. Results of nucleolar organizing region staining. Cancer 71:3114-3118, 1993

29. Schild SE, Nisi K, Scheithauer BW, et al: The results of radiotherapy for ependymomas: the Mayo Clinic experience. Int J Radiat Oncol Biol Phys 42:953-958, 1998

30. Schurmann K, Wallenfang T: Rare sacral space-occupying lesions, their surgical management and reconstructive measures involved. Acta Neurochir 92:106-117, 1988

31. Schweitzer JS, Batzdorf U: Ependymoma of the cauda equina region: diagnosis, treatment, and outcome in 15 patients. Neurosurgery 30:202-207, 1992

32. Scott M: Infiltrating ependymomas of the cauda equina. Treatment by conservative surgery plus radiotherapy. J Neurosurg 41:446-448, 1974

33. Sonneland PR, Scheithauer BW, Onofrio BM: Myxopapillary ependymoma. A clinicopathologic and immunocytochemical study of 77 cases. Cancer 56:883-893, 1985

34. Timmerman W, Bubrick MP: Presacral and postsacral extraspinal ependymoma. Report of a case and review of the literature. Dis Colon Rectum 27:114-119, 1984

35. Waldron JN, LaPerriere NJ, Jaakkimainen L, et al: Spinal cord ependymomas: a retrospective analysis of 59 cases. Int J Radiat Oncol Biol Phys 27:223-229, 1993

36. Wen BC, Hussey DH, Hitchon PW, et al: The role of radiation therapy in the management of ependymomas of the spinal cord. Int J Radiat Oncol Biol Phys 20:781-786, 1991

37. Whitaker SJ, Bessell EM, Ashley SE, et al: Postoperative radiotherapy in the management of spinal cord ependymoma. J Neurosurg 74:720-728, 1991

38. Yucesoy K, Ozer E, Koyuncuoglu M: Parenchymal brain metastasis of a spinal myxopapillary ependymoma after extradural manipulation. Acta Neurochir 143:1071-1072, 2001

Manuscript received September 25, 2003.

Accepted in final form October 10, 2003.

Address reprint requests to: Meic H. Schmidt, M.D., Department of Neurosurgery, University of Utah, 30 North 1900 East, Suite 3B409, Salt Lake City, Utah 84132. email: meic.schmidt@ hsc.utah.edu. 